\title{
An interval observer design for uncertain nonlinear systems based on the T-S fuzzy model
}

\author{
YAMINA MENASRIA, HICHEM BOURAS and NASREDDINE DEBBACHE
}

\begin{abstract}
A new approach to build an interval observer for nonlinear uncertain systems is presented in this paper. Nonlinear systems modeled in the Takagi-Sugeno (T-S) form are studied. A T-S proportional observer is first issued by pole-placement and LMI tools. Secondly, time-varying change of coordinates for each dynamic state estimation error is used to design an interval observer. The system state bounds are then directly deduced.
\end{abstract}

Key words: T-S model, T-S proportional observer, interval observer, time-varying systems.

\section{Introduction}

The problem of state vector estimation is very challenging in control and diagnosis thories for nonlinear systems. It have recently received considerable interest among scientists in various fields and its solution remains expected in many applications. The design of the classical state estimator (observer) is not possible due to presence of uncertainty (parametric or/and signal). A called interval observer, was introduced by [9] to estimate state bounds of biological systems that are subject to parameter uncertainties. Later the framework of interval observers was used and extended for many biological processes [3,21, 15].

Actually, there exist many interval observers proposed for linear systems in continuous and discret times $[13,20,12,7]$. For nonlinear systems, several observer were also proposed in $[14,19,16,18,6,8,5,24]$. By applying similarity transformation, a Hurwitz matrix can be transformed to a Hurwitz and Metzler (cooperative) one. The transformation matrix is constant and real is considered in [18] and it is a solution of the Sylvester equation. In $[13,12]$ the transformation is time-varying.

In this work, we propose the design of an interval observer for nonlinear systems based on the Takagi-Sugeno model with the time-varying approach [12]. The T-S fuzzy model proposed by [22] has been shown to be an universal approximator of nonlinear dynamic systems. It's a piecewise interpolation of several linear or nonlinear models

The authors are with Department of Electronic, Badji Mokhtar University, Po. Box 12, 23000, Annaba, Algeria. The corresponding author is Y. Menasria, e-mail: yamina.menasria@ annaba-univ.org

Received 30.10.2016. Revised 28.3.2017. 
through membership functions. The fuzzy proportional observer cited in [11, 23] is designed but diverges in presence of disturbances. When disturbances are with known distribution, fuzzy unknown input observer introduced by [2] can easily be applied. Fuzzy sliding mode observer studied by [1] works if uncertainties are of known structure. In the case of unknown disturbances but bounded within known bounds, the fuzzy interval observer is a solution.

In the following, an interval observer is designed the T-S systems. Fuzzy interval observer, which is quite an important issue has not been investigated yet. This motivates us to carry out the present work. The design procedure consists in computing proportional observer gains as well as changes of coordinates by multiple time-varying transformations. The main contributions of this paper can be summarized as follows: (i) the fuzzy proportional observer gain matrices are obtained by pole-placement and LMI tools, (ii) time-varying transformation is applied for all local linear models and (iii) sufficient conditions for designing interval observers for T-S systems are given. The rest of the paper is outlined as follows. In Sect. 2, problem formulation and some necessary definitions are given. In Sect. 3, based on time-varying transformation, sufficient conditions for the existence of fuzzy interval observers are established. An example is provided to illustrate the efficiency of the proposed method in Sect. 4. Conclusions are given in Sect. 5.

\section{Problem formulation and preliminaries}

Consider the nonlinear system in the T-S model form:

$$
\left\{\begin{array}{l}
\dot{x}(t)=\sum_{i=1}^{M} \mu_{i}(\xi(t))\left(A_{i} x(t)+B_{i} u(t)\right) \\
y(t)=\sum_{i=1}^{M} \mu_{i}(\xi(t)) C_{i} x(t)
\end{array}\right.
$$

where $M$ is the number of local models function, $x \in \mathfrak{R}^{n}$ is the state vector, $u \in \mathfrak{R}^{p}$ is the input and $y \in \mathfrak{R}^{q}$ the output. Matrices $A_{i}, B_{i} C_{i}$ are constant and the premisse variable $\xi(t)$ can be the control $u(t)$ and/or the state vector $x(t)$.

The membership functions satisfy the following convexity constraints:

$$
\begin{aligned}
& \left\{\begin{array}{l}
\sum_{i=1}^{M} \mu_{i}(\xi(t))=1 \\
0 \leqslant \mu_{i}((\xi)(t)) \leqslant 1
\end{array}\right. \\
& \forall i=1,2 \ldots M
\end{aligned}
$$


The T-S proportional observer is an interpolation of linear proportional observers initiated by [10]. It is given by the following equation:

$$
\left\{\begin{array}{l}
\dot{\hat{x}}(t)=\sum_{i=1}^{M} \mu_{i}(\xi(t))\left(A_{i} \hat{x}(t)+B_{i} u(t)+L_{i}(y(t)-\hat{y}(t))\right. \\
\hat{y}(t)=\sum_{i=1}^{M} \mu_{i}(\xi(t)) C_{i} \hat{x}(t)
\end{array}\right.
$$

The dynamic error state estimation is then:

$$
\dot{e}(t)=\sum_{i=1}^{M} \mu_{i}(\xi(t))\left(A_{i}-L_{i} C_{i}\right) e(t)
$$

The pair $\left(A_{i}, C_{i}\right)$ is detectable for all $i=1 \ldots M$. So, there exist constant matrices $L_{i} \in \mathfrak{R}^{n \times q}$ such that $A_{i}-L_{i} C$ are Hurwitz for all $i=1 \ldots M$. For the sake of simplicity we choose $C_{i}=C$ for all $i=1 \ldots M$ and $L_{i}$ gains are obtained by pole-placement. Global stability is ensured by LMI tools [4].

\section{Nonlinear interval observer design}

Consider the nonlinear uncertain system:

$$
\left\{\begin{array}{l}
\dot{x}(t)=\sum_{i=1}^{M} \mu_{i}(\xi(t))\left(A_{i} x(t)+B_{i} u(t)+\omega_{1 i}(t)\right) \\
y(t)=\sum_{i=1}^{M} \mu_{i}(\xi(t)) C_{i} x(t)+\omega_{2}(t)
\end{array}\right.
$$

where $\omega_{1 i}(t)$, for $i=1 \ldots M$ and $\omega(t)$ are unknown Lipschitz functions with known bounds and the initial condition $x\left(t_{0}\right)=x_{0}$ is assumed to be bounded by two known bounds:

$$
\omega_{1 i}^{-}(t) \leqslant \omega_{1 i}(t) \leqslant \omega_{1 i}^{+}(t)
$$

for all $i=1 \ldots M$ and

$$
\left\{\begin{array}{l}
\omega_{2}^{-}(t) \leqslant \omega_{2}(t) \leqslant \omega_{2}^{+}(t) \\
x_{0}^{-} \leqslant x_{0} \leqslant x_{0}^{+}
\end{array}\right.
$$

The dynamic error state estimation is:

$$
\dot{e}(t)=\sum_{i=1}^{M} \mu_{i}(\xi(t))\left(\left(A_{i}-L_{i} C\right) e(t)\right)+\sum_{i=1}^{M} \mu_{i}(\xi(t))\left(\omega_{1 i}(t)-L_{i} \omega_{2}(t)\right)
$$


Remark 1 For $i=1 \ldots M$, if the corresponding $\left(A_{i}-L_{i} C\right)$ Jordan matrices [17] are not cooperatives (off diagonal entries negative), the system state estimation error (6) can be transformed into cooperative one combining $\mathrm{M}$ linear time-varying change of coordinates.

Theorem 3 The following system:

$$
\left\{\begin{aligned}
\dot{z}^{+}(t) & =\sum_{i=1}^{M} \mu_{i}(\xi(t))\left(G_{i} z^{+}+E_{i}^{+}(t) \varphi_{i}^{+}(t)-E_{i}^{-}(t) \varphi_{i}^{-}(t)\right) \\
\dot{z}^{-}(t) & =\sum_{i=1}^{M} \mu_{i}(\xi(t))\left(G_{i} z^{-}+E_{i}^{+}(t) \varphi_{i}^{-}(t)-E_{i}^{-}(t) \varphi_{i}^{+}(t)\right) \\
e^{+}(t) & =\sum_{i=1}^{M} \mu_{i}(\xi(t))\left(F_{i}^{+}(t) z^{+}(t)-F_{i}^{-}(t) z^{-}(t)\right) \\
e^{-}(t) & =\sum_{i=1}^{M} \mu_{i}(\xi(t))\left(F_{i}^{+}(t) z^{-}(t)-F_{i}^{-}(t) z^{+}(t)\right)
\end{aligned}\right.
$$

where $E_{i}^{+}(t)=\max \left(E_{i}(t), 0\right), E_{i}^{-}(t)=E_{i}^{+}(t)-E_{i}(t)$ and the matrix $F_{i}(t)$ is the inverse of $E_{i}(t)$ with $F_{i}^{+}(t)=\max \left(F_{i}(t), 0\right), F_{i}^{-}(t)=F_{i}^{+}(t)-F_{i}(t)$, is a T-S interval observer of system (6). Disturbances functions $\varphi_{i}^{-}(t)$ and $\varphi_{i}^{+}(t)$ are known bounds of $\varphi_{i}(t)=$ $\omega_{1 i}-L_{i} \omega_{2}$ for $i=1 \ldots M$.

Consequently, the system state bounds are:

$$
\left\{\begin{array}{l}
x^{+}(t)=e^{+}(t)+\hat{x}(t) \\
x^{-}(t)=e^{-}(t)+\hat{x}(t)
\end{array}\right.
$$

Proof We use a time-varying change of coordinate $z(t)=E_{i}(t) e(t)$ for each local model $\dot{e}=\left(A_{i}-L_{i} C\right) e+\varphi_{i}(t)$ and from the convexity constraints of the membership functions $\mu_{i}$ we prove that system (7) is an T-S interval observer of system (6). Globally and from the T-S model:

$$
\dot{z}(t)=\sum_{i=1}^{M} \mu_{i}(\xi(t))\left(G_{i} z(t)+E_{i}(t) \varphi_{i}(t)\right)
$$

let $A_{i}-L_{i} C=\bar{A}_{i}$, then locally, for $i=1 \ldots M$, we have:

$$
\begin{aligned}
& \dot{z}=E_{i}(t) \dot{e}+\dot{E}_{i}(t) e(t) \\
& =E_{i}(t)\left(\bar{A}_{i} e+\varphi_{i}(t)\right)+\left(G_{i} E_{i}(t)-E_{i}(t) \bar{A}_{i}\right) e(t) \\
& =G_{i} E_{i}(t) e(t)+E_{i}(t) \varphi_{i}(t) \\
& =G_{i} z(t)+E_{i}(t) \varphi_{i}(t)
\end{aligned}
$$

The stability of (7) when both $\varphi_{i}^{+}(t)$ and $\varphi_{i}^{-}(t)$ are identically equal to zero is a consequence of the fact that $\bar{A}_{i}$ are Hurwitz for all $t \in \mathfrak{R}$. 
Consider a solution $(z(t), e(t))$ of (7) with known initial conditions $e\left(t_{0}\right)=e_{0}$, $z\left(t_{0}\right)=\left(z^{+}\left(t_{0}\right), z^{-}\left(t_{0}\right)\right)$ and $e_{0}^{+}, e_{0}^{-}$the state error vectors such that:

$$
e_{0}^{-} \leqslant e_{0} \leqslant e_{0}^{+}
$$

Because the entries $E_{i}^{+}$and $E_{i}^{-}$are nonnegative we get:

$$
\left\{\begin{array}{l}
E_{i}^{+}\left(t_{0}\right) e_{0}^{-} \leqslant E_{i}^{+}\left(t_{0}\right) e_{0} \leqslant E_{i}^{+}\left(t_{0}\right) e_{0}^{+} \\
E_{i}^{-}\left(t_{0}\right) e_{0}^{-} \leqslant E_{i}^{-}\left(t_{0}\right) e_{0} \leqslant E_{i}^{-}\left(t_{0}\right) e_{0}^{+}
\end{array}\right.
$$

for all $i=1 \ldots M$. We get:

$$
\left\{\begin{array}{l}
E_{i}^{+}\left(t_{0}\right) e_{0}^{-}-E_{i}^{-}\left(t_{0}\right) e_{0}^{+} \leqslant z\left(t_{0}\right) \\
z\left(t_{0}\right) \leqslant E_{i}^{+}\left(t_{0}\right) e_{0}^{+}-E_{i}^{-}\left(t_{0}\right) e_{0}^{-}
\end{array}\right.
$$

for all $i=1 \ldots M$. From the constraints of the membership functions in (2), we also get:

$$
\left\{\begin{array}{l}
\sum_{i=1}^{M} \mu_{i}\left(\xi\left(t_{0}\right)\right)\left(E_{i}^{+}\left(t_{0}\right) e_{0}^{-}-E_{i}^{-}\left(t_{0}\right) e_{0}^{+}\right) \leqslant z\left(t_{0}\right) \\
z\left(t_{0}\right) \leqslant \sum_{i=1}^{M} \mu_{i}\left(\xi\left(t_{0}\right)\right)\left(E_{i}^{+}\left(t_{0}\right) e_{0}^{+}-E_{i}^{-}\left(t_{0}\right) e_{0}^{-}\right)
\end{array}\right.
$$

Then the initial conditions of the proposed observer are deduced:

$$
\left\{\begin{aligned}
z^{+}\left(t_{0}\right) & =\sum_{i=1}^{M} \mu_{i}\left(\xi\left(t_{0}\right)\right)\left(E_{i}^{+}\left(t_{0}\right) e_{0}^{+}-E_{i}^{-}\left(t_{0}\right) e_{0}^{-}\right) \\
z^{-}\left(t_{0}\right) & =\sum_{i=1}^{M} \mu_{i}\left(\xi\left(t_{0}\right)\right)\left(E_{i}^{+}\left(t_{0}\right) e_{0}^{-}-E_{i}^{-}\left(t_{0}\right) e_{0}^{+}\right)
\end{aligned}\right.
$$

Moreover, for all $i=1 \ldots M$ :

$$
\left\{\begin{array}{l}
E_{i}^{+}(t) \varphi_{i}^{-}(t) \leqslant E_{i}^{+}(t) \varphi_{i}(t) \leqslant E_{i}^{+}(t) \varphi_{i}^{+}(t) \\
E_{i}^{-}(t) \varphi_{i}^{-}(t) \leqslant E_{i}^{-}(t) \varphi_{i}(t) \leqslant E_{i}^{-}(t) \varphi_{i}^{+}(t)
\end{array}\right.
$$

and

$$
\left\{\begin{array}{l}
E_{i}^{+}(t) \varphi_{i}^{-}(t)-E_{i}^{-}(t) \varphi_{i}^{+}(t) \leqslant E_{i}(t) \varphi_{i}(t) \\
E_{i}(t) \varphi_{i}(t) \leqslant E_{i}^{+}(t) \varphi_{i}^{+}(t)-E_{i}^{-}(t) \varphi_{i}^{-}(t)
\end{array}\right.
$$

Since matrices $G_{i}$ are cooperatives for all $i=1 \ldots M$, membership functions $\mu_{i}$ satisfy constraints (2) and inequalities in (8) hold:

$$
\left\{\begin{array}{l}
\sum_{i=1}^{M} \mu_{i}(\xi(t))\left(G_{i} z^{-}(t)+E_{i}^{+}(t) \varphi_{i}^{-}(t)-E_{i}^{-}(t) \varphi_{i}^{+}(t)\right) \\
\leqslant \sum_{i=1}^{M} \mu_{i}(\xi(t))\left(G_{i} z(t)+E_{i}(t) \varphi_{i}(t)\right) \leqslant \\
\sum_{i=1}^{M} \mu_{i}(\xi(t))\left(G_{i} z^{+}(t)+E_{i}^{+}(t) \varphi_{i}^{+}(t)-E_{i}^{-}(t) \varphi_{i}^{-}(t)\right)
\end{array}\right.
$$


and then:

$$
\left\{\begin{array}{l}
\dot{z}^{-}(t) \leqslant \dot{z}(t) \leqslant \dot{z}^{+}(t) \\
z^{-}(t) \leqslant z(t) \leqslant z^{+}(t)
\end{array}\right.
$$

Also and locally for all $i=1 \ldots M$ :

$$
z^{-}(t) \leqslant E_{i}(t) e(t) \leqslant z^{+}(t)
$$

Since the matrices $F_{i}^{+}(t)$ and $F_{i}^{-}(t)$ for all $i=1 \ldots M$ are nonnegative, for all $t \geqslant 0$, we get:

$$
\left\{\begin{array}{l}
F_{i}^{-}(t) z^{-}(t) \leqslant F_{i}^{-}(t) E_{i}(t) e(t) \leqslant F_{i}^{-}(t) z^{+}(t) \\
F_{i}^{+}(t) z^{-}(t) \leqslant F_{i}^{+}(t) E_{i}(t) e(t) \leqslant F_{i}^{+}(t) z^{+}(t)
\end{array}\right.
$$

and

$$
\left\{\begin{array}{l}
F_{i}^{+}(t) z^{-}(t)-F_{i}^{-}(t) z^{+}(t) \leqslant F_{i}(t) E_{i}(t) e(t) \\
F_{i}(t) E_{i}(t) e(t) \leqslant F_{i}^{+}(t) z^{+}(t)-F_{i}^{-}(t) z^{-}(t)
\end{array}\right.
$$

From the fact that, $F_{i}(t)$ are inverse of $E_{i}(t)$, for all $i=1 \ldots M$ following inequalities hold:

$$
\left\{\begin{array}{l}
e(t) \leqslant F_{i}^{+}(t) z^{+}(t)-F_{i}^{-}(t) z^{-}(t) \\
F_{i}^{+}(t) z^{-}(t)-F_{i}^{-}(t) z^{+}(t) \leqslant e(t)
\end{array}\right.
$$

and from the properties of the membership functions in (2) we get:

$$
\left\{\begin{array}{l}
e(t) \leqslant \sum_{i=1}^{M} \mu_{i}(\xi(t))\left(F_{i}^{+}(t) z^{+}(t)-F_{i}^{-}(t) z^{-}(t)\right) \\
\sum_{i=1}^{M} \mu_{i}(\xi(t))\left(F_{i}^{+}(t) z^{-}(t)-F_{i}^{-}(t) z^{+}(t)\right) \leqslant e(t)
\end{array}\right.
$$

Finally, lower and upper bounds for the system states are directly deduced:

$$
\left\{\begin{array}{l}
x^{+}(t)=\sum_{i=1}^{M} \mu_{i}(\xi(t))\left(F_{i}^{+}(t) z^{+}(t)-F_{i}^{-}(t) z^{-}(t)\right)+\hat{x}(t) \\
x^{-}(t)=\sum_{i=1}^{M} \mu_{i}(\xi(t))\left(F_{i}^{+}(t) z^{-}(t)-F_{i}^{-}(t) z^{+}(t)\right)+\hat{x}(t)
\end{array}\right.
$$




\section{Simulation}

Let us consider the T-S system with two local models $(M=2)$ :

$$
\left\{\begin{array}{l}
A_{1}=\left[\begin{array}{ccc}
-2 & -0.2 & 0.4 \\
5 & -1 & 2 \\
3 & -1 & -2
\end{array}\right] ; B_{1}=\left[\begin{array}{c}
1 \\
-1 \\
2
\end{array}\right] ; \\
C_{1}=\left[\begin{array}{lll}
0 & 1 & 1
\end{array}\right] . \\
A_{2}=\left[\begin{array}{ccc}
-2 & 0 & 1 \\
1 & -2 & 3 \\
0 & -0.2 & -1
\end{array}\right] ; B_{2}=\left[\begin{array}{c}
-2 \\
1 \\
-1
\end{array}\right] ; \\
C_{2}=C_{1} .
\end{array}\right.
$$

The pairs $\left(A_{1}, C_{1}\right)$ and $\left(A_{2}, C_{2}\right)$ are detectables and the T-S proportional observer gains $\left(L_{1}, L_{2}\right)$ are calculated by pole-placement in a stable complex plane region using LMIs. The two state estimation error matrices are Hurwitz and each one has two complex conjugate and one real eigen-values. Consequently, the corresponding Jordan matrices (9) are not cooperatives.

$$
\left\{\begin{array}{c}
J_{1}=\left[\begin{array}{ccc}
-1.8641 & 0 & 0 \\
0 & -2.1082 & -2.6595 \\
0 & 2.6595 & -2.1082
\end{array}\right] \\
J_{2}=\left[\begin{array}{ccc}
-2.2162 & 0 & 0 \\
0 & -2.433 & -1.1521 \\
0 & 1.1521 & -2.433
\end{array}\right]
\end{array}\right.
$$

The system state estimation error must be transformed into cooperative one using two linear time-varying change of coordinates. In Fig. 1, the input $u(t)$ is variable on its entire range $[-1,1]$ in order to excite all local modes. It is also chosen the premisse variable $\xi(t)$ for the T-S system. Fig. 2 illustrates two membership functions that satisfy the convexity criterion (2) at each time. State and output disturbances $\left(\omega_{11}(t), \omega_{12}(t)\right.$ and $\left.\omega_{2}(t)\right)$ are choosen uniformly distributed noise respectively in interval: $[-0.5,+0.5],[-0.5,+0.5]$ and $[-1,+1]$. Like is shown in Figure 3 , the states $x_{1}, x_{2}$ and $x_{3}$ remain inside the interval $\left[x_{i_{i n f}}(t), x_{i_{\text {sup }}}(t)\right]$ respctively for $i=1,2,3$. 


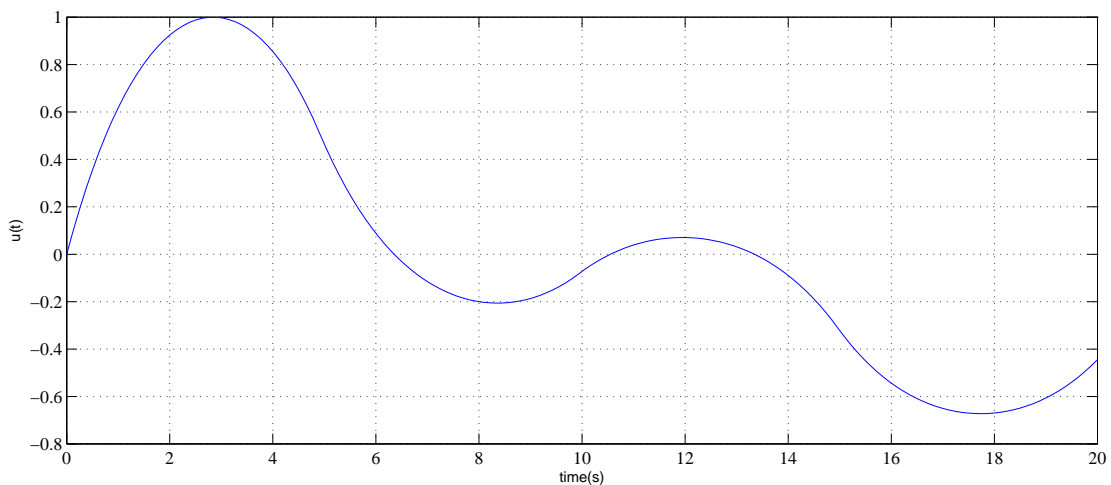

Figure 1: The input signal

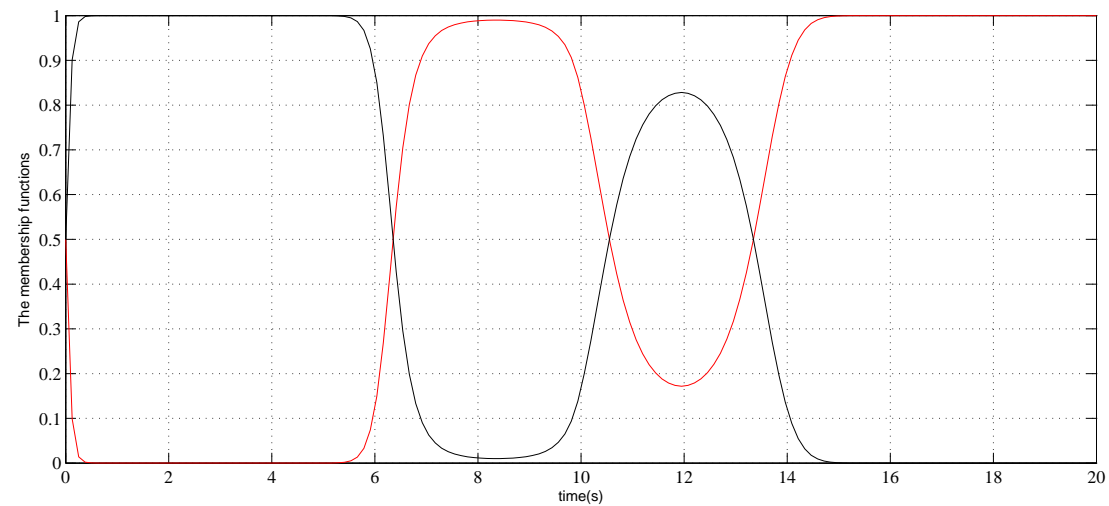

Figure 2: The membership functions: $\mu_{1}$ in red line and $\mu_{2}$ in black line

\section{Conclusions}

Based on interval analysis, which we believe to be an extremely promising approach for the investigation of the properties of nonlinear systems, a guaranteed technique for nonlinear state estimation in a bounded error context have been presented. In First, the fuzzy proportional observer is built without uncertainties. Then Pole-placement ensures that state estimation error matrices are Hurwitz but rarely cooperatives. Finally, time-varying change of coordinates approach changes T-S error state estimation system with disturbances into cooperative one. By knowing initial state interval, T-S interval observer for error state estimation system is designed. From error state estimation bounds, system states bounds are deduced at each time. The fuzzy interval observer proposed in this paper can be applied to several practical systems with unknown disturbances but bounded within known bounds like waste water treatment plants. 

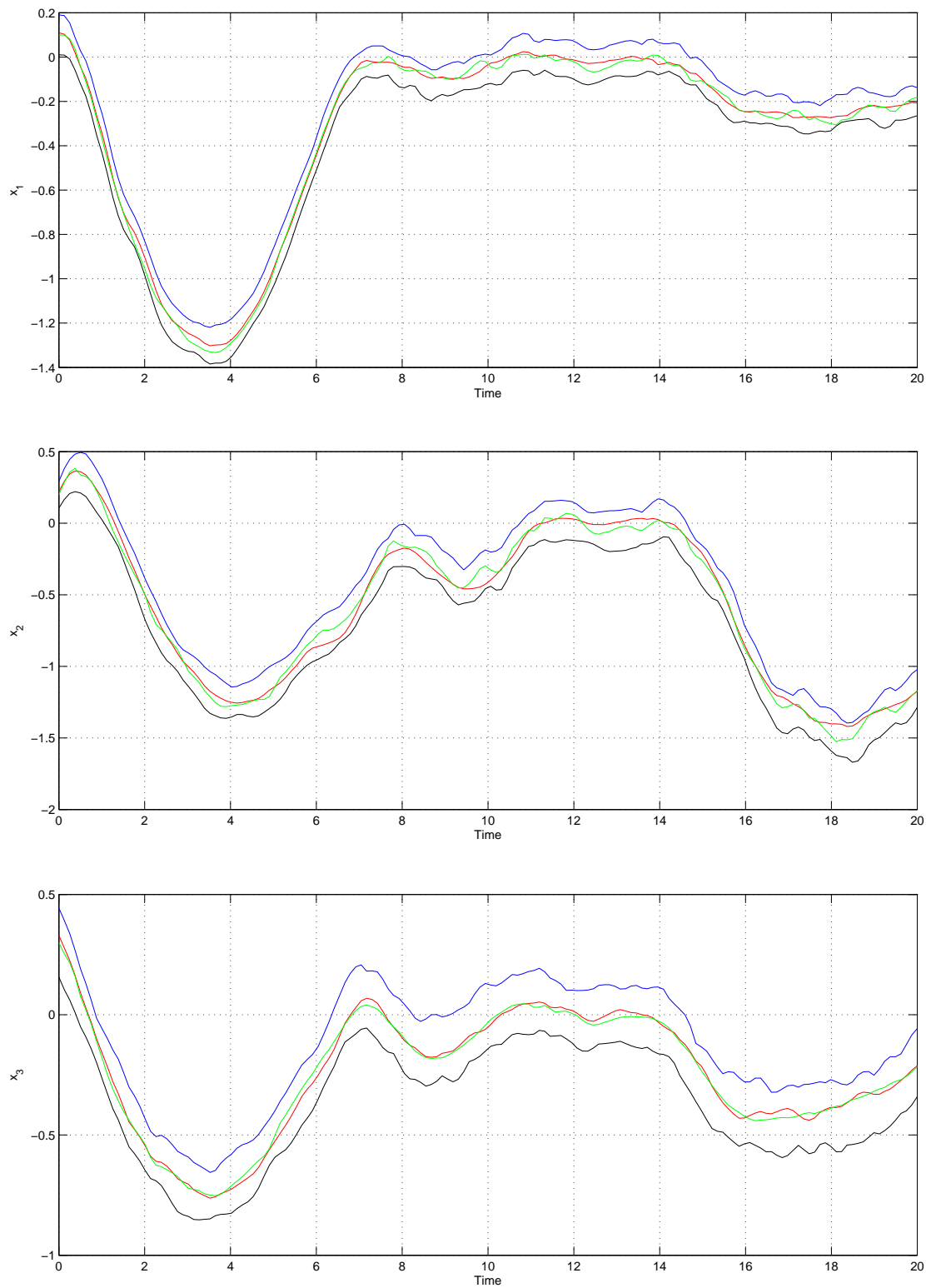

Figure 3: State $x_{1}, x_{2}$ and $x_{3}$ bounds in blue and black lines with the Luenberger observer (green lines) and comparison with the real state (red line). 


\section{References}

[1] A. Akhenak, M. Chadli, J. Ragot and D. MaQuin: Design of robust fuzzy observer for uncertain Takagi-Sugeno models. IEEE Int. Conf. on Fuzzy Systems, Budapest, Hungary, (2004).

[2] A. Akhenak, M. Chadli, J. Ragot and D. Maquin: State estimation via multiple observer with unknown input: Application to the three tank system. 5th IFAC Symp. on Fault Detection Supervision and Safety for Technical Processes, Washington, USA, (2003).

[3] O. Bernard and J.L. Gouze: Closed loop observers bundle for uncertain biotechnological models. J. of Process Control, 14, (2004), 765-774.

[4] S. Boyd, L. Ghaoui, E. Feron and V. Balakrishnan: Linear Matrix Inequalities in System and Control Theory. SIAM, Philadelphia, 1994.

[5] S. Chebotarev, D. Efimov, T. Raissi and A. Zolghadri: On interval observer design for a class of continuous-time LPV systems. IFAC Nolcos, Toulouse, France, (2013).

[6] D. Efimov, L. Fridman, T. Raissi, A. Zolghadri and R. Seydoud: Interval estimation for LPV systems applying high order sliding mode techniques. Automatica, 48, (2012), 2365-2371.

[7] D. Efimov, W. Perruquetti, T. Raissi and A. Zolghadri: On interval observer design for time-invariant discrete-time systems. European Control Conf., Zurich, Switzerland, (2013).

[8] D. Efimov, T. Raissi, A. Chebotarev and A. Zolghadri: Interval state observer for nonlinear time varying systems. Automatica, 49, (2013), 200-205.

[9] J.L. GouZE, A. RAPAPORT and Z. HADJ-SADOK: Interval observers for uncertain biological systems. Ecological Modelling, 133, (2000), 45-56.

[10] D.G. LUEnberger: Observers of multivariable systems. IEEE Trans. on Automatic Control, 11, (1966), 190-197.

[11] X.J. MA, Z.Q. Sun and Y.Y. HE: Analysis and design of fuzzy controller and fuzzy observer. IEEE Trans. on Fuzzy Systems, 6, (1998), 41-51.

[12] F. MAZENC and O. BERNARD: Interval observers for linear time-invarariant systems with disturbances. Automatica, 47, (2011), 140-147,

[13] F. MAZENC and O. BernARD: Asymptotically stable interval observers for planar systems with complex poles. IEEE Trans. on Automatic Control, 55, (2010), 523527. 
[14] M. MoISAN and O. BERNARD: Robust interval observers for uncertain chaotic systems. 45th IEEE Conf. on Decision and Control, San Diego, USA, (2006).

[15] M. Moisan, O. Bernard and J.L. Gouze: Near optimal interval observers bundle for uncertain bioreactors. Automatica, 45, (2009), 291-295.

[16] |sc M. Moisan and O. Bernard: Robust interval observers for global Lipschitz uncertain chaotic systems. Systems and Control Letters, 59, (2010), 687-694.

[17] L. Perko: Differential Equations and Dynamical Systems. $3^{\text {rd }}$ Edition. Springer, 2000.

[18] T. RAISSI, D. EFIMOV and A. ZolghadRI: Interval state estimation for a class of nonlinear systems. IEEE Trans. on Automatic Control, 57, (2012), 260-265.

[19] T. RAISSI, G. VidEAU and A. ZolghadRI: Interval observer design for consistency checks of nonlinear continuous-time systems. Automatica, 46, (2010), 518527.

[20] M.A. RAMI, J. JORDAN and M. SCHONLEIN: Interval observers for linear systems with time-varying delays. Int. Symp.on Mathematical Theory of Networks and Systems, Budapest, Hungary, (2010).

[21] A. RAPAPORT and D. Dochain: Interval observers for biochemical processes with uncertain kinetics and inputs. Mathematical Biosciences, 193, (2005), 235253.

[22] T. TAKAGI and M. SugENO: Fuzzy identification of systems and its applications to modeling and control. IEEE Trans. on Systems Man and Cybernetic, 15, (1985), 116-132.

[23] H.O. WANG and K. Tanaka: Fuzzy control systems design and analysis. John Wiley \& Sons., 2001.

[24] G. Zheng, D. Efimov and W. Perruquetti: Design of interval observer for a class of uncertain unobservable nonlinear systems. Automatica, 63, (2016), 167174. 